\title{
Aquifer design for freshwater storage and recovery in artificial islands and coastal expansions
}

\author{
Marloes van Ginkel
}

Keywords Artificial recharge · Coastal aquifers $\cdot$ Hydraulic properties $\cdot$ Artificial Islands

\section{Introduction}

Artificial recharge of water to an aquifer for later recovery and use, otherwise known as artificial storage and recovery (ASR; Pyne 1995), is nowadays widely applied for seasonal or emergency water storage. Therefore, one would expect a lot of thought to be devoted to what the optimal aquifer conditions for ASR would be, but this does not appear to be the case.

In some situations local hydrogeology may impact the selection of ASR sites; however, according to Pyne (1995), this is the exception rather than the rule since ASR wells are usually located where they provide the greatest benefit to the water utility or agency. This location is often in the vicinity of the supply area to reduce the costs and time of transportation. At that specific site, one deals with the existing local hydrogeology through well design and construction (e.g., Maliva and Missimer 2010; Zuurbier et al. 2014) and operation (Ward et al. 2009; Bakker 2010). In line with Pyne's field experience, Lowry and Anderson (2006) distinguished physical properties of the aquifer and operational factors that control the recovery efficiency of ASR. Properties like porosity, hydraulic conductivity, aquifer thickness and density of native water, as well as quality, are regarded as predefined site-specific conditions, while operational factors such

Received: 1 September 2014 / Accepted: 4 March 2015

Published online: 21 April 2015

(C) The Author(s) 2015. This article is published with open access at Springerlink.com

M. Ginkel $(\square)$

Faculty of Civil Engineering and Geosciences, Water Resources Section, Delft University of Technology, PO Box 50482600 GA, Delft, The Netherlands

e-mail: marloes.vanginkel@gmail.com

M. Ginkel

Royal Haskoning DHV, PO Box 85203009 AM, Rotterdam, The Netherlands as injected volume, location of injection and recovery wells, recharge and recovery rates and storage duration, can be changed at the wellhead by the operator to optimize the ASR system.

The most relevant research determining optimal aquifer conditions for ASR focused on the influence of aquifer properties on the recovery efficiency of ASR wells. These studies are, firstly, comparisons between ASR sites, such as the studies executed by e.g., Merritt (1986), Dillon et al. (2006), Lowry and Anderson (2006) and Misut and Voss (2007). On the other hand, there are theoretical considerations regarding dimensionless parameter groups in analytical solutions, as described by e.g., Esmail and Kimbler (1967), Ward et al. (2007, 2008, 2009) and Bakker (2010). The general outcomes of these studies indicate that porosity, hydraulic conductivity, vertical anisotropy, dispersivity, density of native water versus that of the injected water, and the thickness of the aquifer all influence the recovery efficiency. This makes sense because these aquifer properties determine the degree of the underlying recovery efficiency processes of mixing and density stratification.

The question of which aquifer conditions are preferable for ASR is usually not relevant in practice because the costs of the required earth displacement and construction works are so high that they generally outweigh the economic benefits of ASR systems. However, the situation is different in the case of coastal expansions and artificial islands that are currently being constructed worldwide. The most well-known examples are probably the Palms and the islands of the World Archipelago in Dubai (UAE) with a mainly touristic purpose. Artificial islands are also constructed for industrial development such as Maasvlakte II in the Port of Rotterdam, the Netherlands. Recently, there is a tendency in densely populated delta cities for urban expansion toward the ocean; examples are Eko Atlantic in the City of Lagos, Nigeria, and the planned land reclamations in Jakarta Bay, Indonesia. Aquifers are, in fact, created in these projects and their conditions can be optimized for specific ASR applications. Specific sediment types may be chosen and different dredging techniques can be applied to create the "optimal" aquifer conditions for recharge and recovery in terms of porosity, conductivity, anisotropy, and dispersivity to control mixing processes and density stratification. 
Three artificial aquifer designs are discussed in this essay. The aim of the essay is to make readers aware that aquifers are created while constructing artificial islands and that this provides opportunities for ASR. This perspective may change the way we look at the optimal hydraulic properties of artificial islands and coastal expansions.

\section{Artificial aquifer design 1}

Considering what optimal conditions for ASR would be, one might initially think of a design as presented in Fig. 1 in which the stored water is completely separated from the surrounding groundwater system. In artificial aquifer design 1 , vertical walls of impermeable material such as clay or sheet piles along the storage zone and a confining layer at the bottom prevent interaction with lower quality ambient water or water with a different density. Water infiltrates at the top and seeps through the storage zone. The water is recovered through horizontal wells from a layer of gravel at the bottom. This layer has a relatively high hydraulic conductivity, thus allowing an evenly distributed lowering of the water table, which results in a relatively quick recovery, also from the outer regions of the storage zone.

On further consideration, however, the continuous alteration between anaerobic and aerobic conditions could well result in internal contamination of e.g., iron and manganese precipitates resulting in clogging. Whether these reactions will occur in practice, is highly dependent on the aquifer material and nutrients in the injection water. The wells at the bottom of the storage zone are also practically inaccessible for maintenance and replacement. Finally, the design is expected to be costly and it will be difficult to completely guarantee that the vertical walls and the confining layer at the bottom will be sufficiently impermeable. Seepage of poor-quality water through the impervious walls could pollute the stored water with little chance to clean it other than complete replacement of the fill.

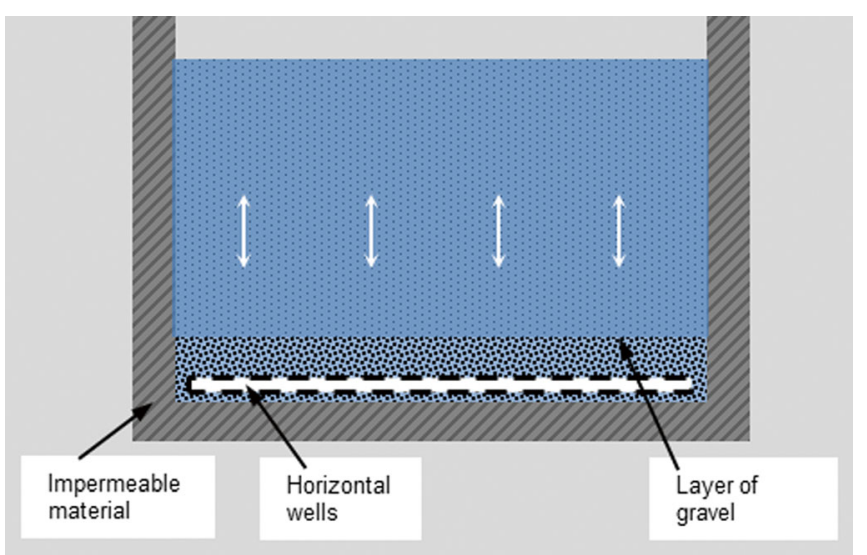

Fig. 1 Artificial aquifer design 1: the storage zone is completely separated from the surrounding groundwater system through vertical walls and a confining bottom layer

Hydrogeology Journal (2015) 23: 615-618
It thus appears that complete separation of the storage zone from its environment is not as optimal as perhaps initially thought. Solutions to clogging are, firstly, the prevention of alternating anaerobic and aerobic conditions, secondly, the choice of aquifer material and the separation of different sediment types, and, thirdly, regular flushing of the system. The latter requires the stored water to be in open communication with the surrounding groundwater system. The construction of a partly open ASR system will be simpler and cheaper compared to artificial aquifer design 1 . However, the injected water will inevitably come into contact with native groundwater, which is generally saline water in coastal expansions or artificial islands. Mixing and density stratification will occur and the question arises as to how the aquifer should be designed such that injected water can be kept both in place and separated from ambient water. In the remainder of this essay, that question is discussed with the help of two potential artificial aquifer designs.

\section{Artificial aquifer design 2}

Consider an unconfined saline aquifer of which a certain part is surrounded by vertical impermeable walls that partly penetrate the aquifer (Fig. 2). When freshwater is infiltrated between the walls, the density difference between the two water types causes the lighter freshwater to float on top of denser saline groundwater; the mixing zone separates the two fluids. Usually, the problem with freshwater injected into aquifers containing denser saltwater is that the freshwater volume tends to float upward to the top of the aquifer and spreads out, where it is impossible to recover at a later stage. In artificial aquifer design 2, the vertical walls obstruct the freshwater volume from expanding radially (Van Ginkel et al. 2014). Such walls were already suggested by e.g., Anwar (1983) and Luyun et al. (2011) as a measure to prevent saltwater intrusion in coastal aquifers.

The interface between injected freshwater and native saline water will gradually turn into a transition zone

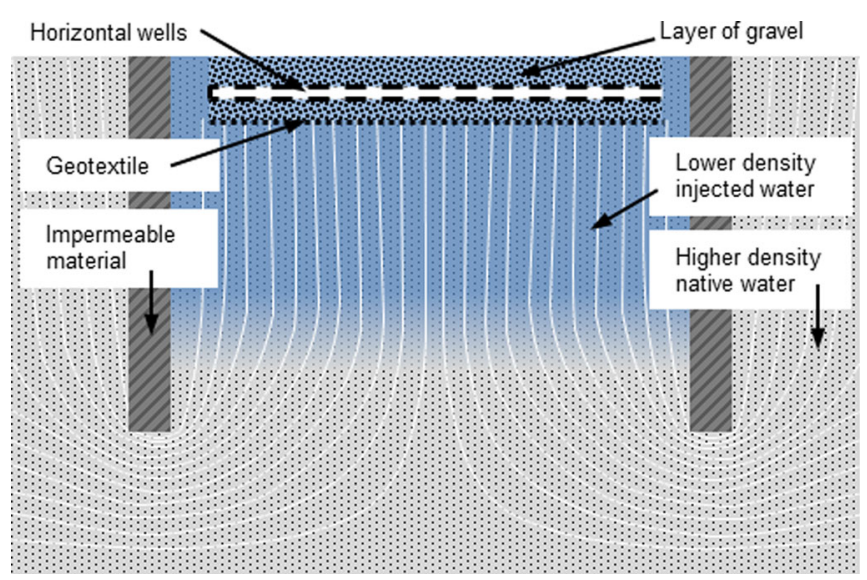

Fig. 2 Artificial aquifer design 2: ASR in a saline aquifer bounded by partly penetrating impermeable walls (the white lines indicate stream lines during injection and recovery) 
between the two water types (e.g., Esmail and Kimbler 1967; Verruijt 1971). The amount of mixing is theoretically controlled by the longitudinal and transverse dispersivities, and the flow velocity, as well as molecular diffusion. Dispersivity increases significantly with the heterogeneity of the aquifer material. The storage zone should, thus, consist of homogeneous fine sand to minimize mixing.

Conditions should be such that freshwater can be recovered quickly with the interface staying more or less horizontal. Freshwater is, therefore, preferably recovered by horizontal wells at the top of the aquifer. Horizontal wells in a layer of gravel at the top of the aquifer ensure small head gradients during recovery, thus minimizing saltwater upconing. The thickness of the layer of gravel and the required recovery rates should be balanced to decrease the risk of wells running dry. A geotextile between the layer of gravel and the underlying sand prevents fines from being washed into the gravel layer, where they might otherwise cause clogging. During pumping, the vertical flow velocity will unevenly be distributed over the width of the storage zone. Especially notice that the flow rates will be considerably higher along the edges than in the middle due to contraction of stream lines below the vertical wall (Fig. 2). The fluctuations in flow velocity may be counteracted by spatially adjusting the grain size of the sediment within the storage area. While the required grain-size distribution can readily be modelled, methods to actually realize this have yet to be researched and developed.

The placement of material during dredging works may not be as accurate as theoretically desired, potentially causing unexpected and unknown spatial variations in the characteristics of the artificial aquifer. This could result in preferential flow paths or flow zones, which may substantially affect flow and mixing (e.g., Fiori and Jankovic 2012; Dagan et al. 2013) and may lead to advection-induced vertical fingering. The layer of gravel at the top should preferably be constructed after the in situ hydraulic distribution of the lower layer has been determined, so that the properties of the gravel layer can be tailored to compensate for spatial variations of the conductivity in the underlying sand. Effective in situ spatial hydraulic conductivity testing is another technology to be developed.

Leakage through the enclosing walls may occur due to construction errors and phenomena such as rabbit holes and wormholes, cracks caused by uneven settlement, and desiccation of clays. While during storage periods the density difference between freshwater and the surrounding saline groundwater would force outward leakage, inflow of saline groundwater would occur during recovery, when the head in the storage zone is low. Some leaked-in saline water may, thus, be present after a recovery period, which fortunately tends to sink downward during storage periods due to its higher density. This process may be enhanced by active flushing.

\section{Artificial aquifer design 3}

Artificial aquifer design 3 may be preferred where the ocean floor consists of clay, as is often the case in deltas (Fig. 3). This clay restricts the depth of the storage zone (Tijs 2014). In such shallow and extended artificial aquifers, freshwater is best injected and recovered by multiple fully penetrating wells. When using such systems of individual wells, the size of the stored volume associated with each well is limited by the time required to inject and extract the water. Artificial aquifer design 3 is, therefore, restricted to relatively small storage volumes per well.

An initial vertical interface between two fluids with different densities, as it develops after a vertical ASR-well starts injecting, will rotate as has often been demonstrated, e.g., Bakker et al. (2004) and Van Ginkel et al. (2014). As time passes, the two fluids stratify with the denser fluid spreading along the bottom and the lighter fluid accumulating along the top of the aquifer. It is desirable to limit buoyancy-induced flow as much as possible in an attempt to prevent this density stratification. Ideally, the interface would remain vertical. This requires only horizontal flow, i.e., zero vertical flow. In any case, the vertical anisotropy should be as large as possible. Kumar and Kimbler (1970) and Ward et al. (2008) already mentioned that layering improves the recovery efficiency where the individual layers have identical properties and there is no cross flow between them. The segregation of coarser and finer particles that always occurs during submerged settling of dredged material may be exploited and optimized to obtain such desired aquifer properties. This requires a layer-after-layer build-up of the aquifer where small layers of sand must be distributed evenly over the entire width of the storage zone and then left sufficient time to settle. Although this technique is more time consuming and therefore more expensive than regular dredging, it is often applied on clayey ocean floors for geotechnical reasons.

Artificial aquifer design 3 consists of many thin layers, each with a grain size that varies from coarse to fine vertically. The design inevitably risks entrainment of fines into the well, which may cause all kind of problems such

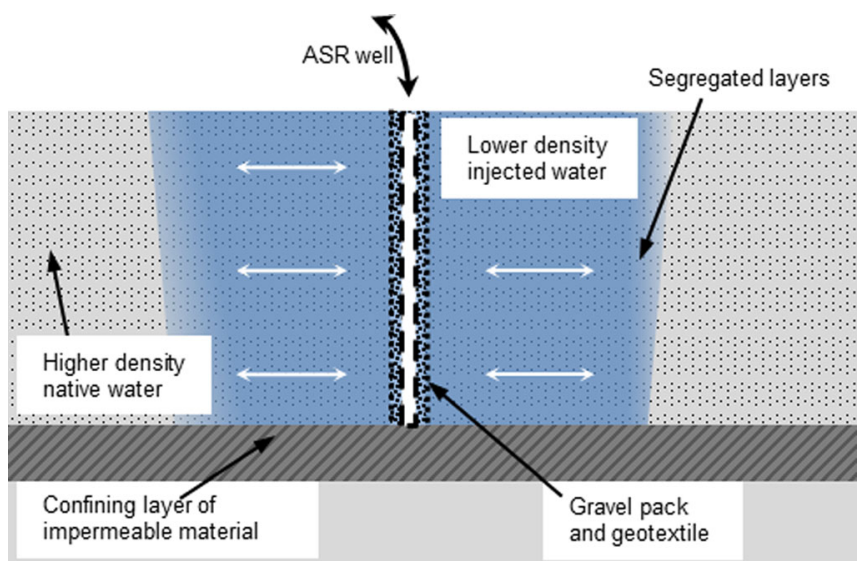

Fig. 3 Artificial aquifer design 3: ASR in a saline aquifer with segregated layers 
as clogging, braising of pump impellers and damage of the aquifer itself by loss of particles. The design of the gravel pack and screen slot thus requires special attention and a geotextile around the gravel pack of each well may be required to prevent fines from being washed into the gravel pack and the well.

\section{Discussion}

The objective of this essay was to raise awareness that aquifers are nowadays created in coastal expansion projects and during construction of artificial islands, whereby optimization of their physical properties for ASR becomes possible. Dynamic water storage in such aquifers will likely add value to the future development on the island. While this essay points to necessary research and development, the general requirements of different designs of artificial aquifers for freshwater storage and recovery have also been outlined.

The focus of this essay was on small-scale ASR in coastal expansions and artificial islands. The take-home message is that the entire water system should be taken into account when designing new developments from scratch, in which ASR could naturally take part.

The biggest challenge is probably in the linking of hydrologists and dredging engineers with each other. Dredging techniques are well studied, but because engineers concerned with the design and construction of islands are unaware of future water demands on these newly constructed lands and the potentials of ASR, they do not consider multifunctional use of the subsurface for ASR. Traditionally, dredging engineers focus on the lowest cost of construction and do not have direct responsibility for water supply for the future development on the island. Hydrologists and water engineers appear later and have then to deal with the already constructed island. The design of coastal expansion and artificial islands at present seldom takes into account optimization for future ASR in the newly created aquifer. It is hoped that this essay serves to stimulate the discussion and will encourage cooperation between dredging engineers and hydrologists to develop this new field.

Acknowledgements This essay was originally inspired by conversations of the author with Dr. C.I. Voss at the Salt Water Intrusion Meeting 2014 in Husum, Germany.

Open Access This article is distributed under the terms of the Creative Commons Attribution License which permits any use, distribution, and reproduction in any medium, provided the original author(s) and the source are credited.

\section{References}

Anwar HO (1983) The effect of a subsurface barrier on the conservation of freshwater in coastal aquifers. Water Resour Res 17(10):1257-1265
Bakker M (2010) Radial Dupuit interface flow to assess the aquifer storage and recovery potential of saltwater aquifers. Hydrogeol J 18:107-115. doi:10.1007/s10040-009-0508-1

Bakker M, Oude Essink GHP, Langevin CD (2004) The rotating movement of three immiscible fluids: a benchmark problem. J Hydrol 287:270-278. doi:10.1016/j.jhydrol.2003.10.007

Dagan G, Fiori A, Jankovic I (2013) Upscaling of flow in heterogeneous porous formations: critical examination and issues of principle. Adv Water Resour 51:67-85. doi:10.1016/ j.advwatres.2011.12.017

Dillon PJ, Ravelic P, Toze S, Rinck-Pfeiffer S, Martin R, Knapton A, Pidsley D (2006) Role of aquifer storage in water reuse. Desal 188:123-134. doi:10.1016/j.desal.2005.04.109

Esmail OJ, Kimbler OK (1967) Investigation of the technical feasibility of storing fresh water in saline aquifers. Water Resour Res 3(3):686-695

Fiori A, Jankovic I (2012) On preferential flow channeling and connectivity in heterogeneous porous formations. Math Geosci 44:133-145. doi:10.1007/s11004-011-9365-2

Kumar A, Kimbler OK (1970) Effect of dispersion, gravitational segregation, and formation stratification on the recovery of freshwater stored in saline aquifers. Water Resour Res 6(6):1689-1700

Lowry CS, Anderson MP (2006) An assessment of aquifer storage recovery using groundwater flow models. Ground Water 44(5):661-667. doi:10.1111/j.1745-6584.2006.00237.x

Luyun R Jr, Momii K, Nakagawa K (2011) Effects of recharge wells and flow barriers on seawater intrusion. Ground Water 49(2):239-249. doi:10.1111/j.1745-6584.2010.00719.x

Maliva RG, Missimer TM (2010) Aquifer storage and recovery and managed aquifer recharge using wells: planning, hydrogeology, design and operation. Methods in Water Resources Evaluation Series no. 2. Schlumberger, Houston, TX

Merritt ML (1986) Recovering fresh water stored in saline limestone aquifers. Ground Water 24(4):516-529

Misut PE, Voss CI (2007) Freshwater-saltwater transition zone movement during aquifer storage and recovery cycles in Brooklyn and Queens, New York City, USA. J Hydrol 337:87-103. doi:10.1016/j.jhydrol.2007.01.035

Pyne RDG (1995) Aquifer storage recovery: a guide to groundwater recharge through wells. ASR Systems, Gainesville, FL

Tijs M (2014) Subsurface freshwater storage and recovery in artificial islands, case study of Pluit City Land Reclamation Jakarta, Indonesia. MSc Thesis, Delft University of Technology, The Netherlands

Van Ginkel M, Olsthoorn TN, Bakker M (2014) A new operational paradigm for small-scale ASR in saline aquifers. Ground Water 52:685-693. doi:10.1111/gwat.12113

Verruijt A (1971) Steady dispersion across an interface in a porous medium. J Hydrol 14:337-347

Ward JD, Simmons CT, Dillon PJ (2007) A theoretical analysis of mixed convection in aquifer storage and recovery: how important are density effects? J Hydrol 343:169-186. doi:10.1016/j.jhydrol.2007.06.011

Ward JD, Simmons CT, Dillon PJ (2008) Variable-density modelling of multiple-cycle aquifer storage and recovery (ASR): importance of anisotropy and layered heterogeneity in brackish aquifers. J Hydrol 356:93-105. doi:10.1016/ j.jhydrol.2008.04.012

Ward JD, Simmons CT, Dillon PJ, Pavelic P (2009) Integrated assessment of lateral flow, density effects, and dispersion in aquifer storage and recovery. J Hydrol 370:83-99. doi:10.1016/ j.hydrol.2009.02.055

Zuurbier KG, Zaadnoordijk WJ, Stuyfzand PJ (2014) How multiple partially penetrating wells improve the freshwater recovery of coastal aquifer storage and recovery (ASR) systems: a field and modeling study. J Hydrol 509:430-441. doi:10.1016/ j.jhydrol.2013.11.057 